\title{
Pesticide application with reduced flow
}

Two articles which will have a very great impact are present in Fruits vol. 13 (3-4); both relate to pesticide applications with reduced flow in tropical fruit crops, a topic which has already been explored before.

The first document [Fruits vol. 13 (3)], entitled Attempt at forecasting Cercospora attacks in Guadeloupe, written by Guyot and Cuillé, can be regarded as a founder of the forecasting techniques for the control of banana Sigatoka diseases: 15 years later, Ganry and Meyer will use it as a strong base for building up the forecasting system, which allowed an effective and very great reduction in the quantities of fungicide applied and their polluting effect. This forecasting system is always used in Africa, in the Caribbean and in Brazil, to quote only some places where the environmental impact of the control is strongly reduced compared with other production areas. In this article, an action curve of the temperature on mycelium development was published in particular, which constituted one of the bases of the forecasting system. The authors specified that the results deserved "to be verified and included under different ecological conditions".

It is exactly what was done without any delay in Cameroon; this later study is presented in an article in the next issue [Fruits vol. 13 (4)] under the title "A control campaign against Sigatoka disease in Cameroon" written by Merle, Cuillé and de Laroussilhe. This paper, much documented, shows very good transposability of the preceding results, which will be largely verified thereafter.

Dr. Jacky Ganry Chairman of the Section on Tropical and Subtropical Fruits

ISHS

\section{Traitements pesticides à débit réduit}

Deux articles qui auront une portée très importante figurent dans Fruits vol. 13 (3-4) ; tous deux concernent les traitements pesticides à débit réduit en culture fruitière tropicale, thème qui a déjà été exploré antérieurement.

Le premier document [Fruits vol. 13 (3)], intitulé Essai de prévision des attaques de Cercospora en Guadeloupe, écrit par Guyot et Cuillé, peut être considéré comme fondateur des techniques d'avertissement dans la lutte contre les cercosporioses du bananier : c'est à partir de lui que, 15 ans plus tard, Ganry et Meyer construiront le système d'avertissement qui a permis de réduire très fortement et efficacement les quantités de fongicide appliquées, ainsi que leur effet polluant. Ce système d'avertissement est toujours utilisé en Afrique, aux Antilles, au Brésil, pour ne citer que quelques lieux où l'impact environnemental de la lutte est de ce fait fortement réduit par rapport aux autres zones de production. Dans cet article, paraissait notamment une courbe d'action de la température sur le développement du mycélium, qui a constitué une des bases du système d'avertissement. Les auteurs précisaient que les résultats méritaient " d'être vérifiés et repris dans des conditions écologiques différentes".

C'est exactement ce qui fut fait sans tarder au Cameroun ; cette étude est présentée dans l'article du numéro suivant [Fruits vol. 13 (4)] sous le titre Une campagne de lutte contre Cercospora au Cameroun, rédigé par Merle, Cuillé et de Laroussilhe. Cet article très documenté montre la très bonne transposabilité des résultats précédents, ce qui sera largement vérifié par la suite.

Dr. Jacky Ganry

Président de la section Fruits tropicaux et subtropicaux ISHS 